\title{
A special issue on CCN proteins and cancer
}

\author{
B. Perbal ${ }^{1}$
}

Received: 29 July 2016 / Accepted: 29 July 2016 / Published online: 5 August 2016

(C) The International CCN Society 2016

With this special issue we celebrate 25 years of publishing in the field of CCN protein biology. Since the very first publications reporting the existence of these proteins, strong relationships have been established between the disregulation in the expression of $\mathrm{CCN}$ proteins and the development of cancer.

The existence of positive and negative regulators of cell proliferation and differentiation exibited by the members that constitute the $\mathrm{CCN}$ family soon appeared to be a salient feature of this group of structurally related proteins, and raised challenging questions regarding their specific role in tumor initiation, promotion and progression. Some of the family members have been shown to increase the metastatic potential of tumor cells whereas others behave as tumor suppressors and/or antimetastatic agents.

The impact of CCN protein biology on tumor cell microenvironment has been documented, and constitutes an important area of research (see H. Yeger and B. Perbal in this issue).

To celebrate the recent assignment to JCCS of a noteworthy IF (3.123 in 2015), we have invited several colleagues involved in cancer research to review specific aspects of this important disease. We have also taken the opportunity of recognizing the 25th CCN publishing anniversary to evaluate the impact of the $\mathrm{CCN}$ field through an analysis of the number of articles published since the discovery of the first members of the CCN family.

Among the vast number of articles dealing with different aspects of $\mathrm{CCN}$ protein biology, an important number (172

\section{B. Perbal}

bperbal@gmail.com

1 Université Côte d'Azur, CNRS, GREDEG, Nice France and International CCN Society, Paris, France out of 586 on cancer) have been published on breast cancer over the past decades (see Perbal A. \& Perbal B. in this issue). C. Kleer reviews the powerful effects of microenvironment on breast tumor progression and discusses potential specific therapeutic approaches for the near future that would capitalize on the functions of various $\mathrm{CCN}$ proteins. Breast cancers account for $16 \%$ of all female cancers and nearly $23 \%$ of invasive cancers in women worldwide. Each year, according to the National Cancer Institute (NCI), more than 230,000 breast cancers are reported in females, and a little more than 2000 in males. Breast cancer accounts for $18 \%$ of all cancer deaths worldwide. The expectations for 2016 are 240,000 new cases of which 40,000 will be fatal. The strong support for breast cancer research was illustrated by the recent announcement in the July 19th University of Southern California (USC) news, that a grant of $\$ 12$ million was awarded to researchers of the Keck School of Medicine at USC whose program aims at a better understanding of the biological and genetic features that lead African American women to develop more agressive tumors and with a more rapid fatal outcome. ${ }^{1}$

In their review on the contribution of various $\mathrm{CCN}$ proteins to the development of pancreatic cancer, S. Banerjee et al. also focus on potential therapeutic approaches for this common human cancer with a 5-year survival rate of less than $5 \%$ after diagnosis and treatment,. According to the American Cancer Society, 53,000 individuals (about $50 \%$ of males and females) will be diagnosed with pancreatic cancers in 2016 with approximately 40,000 expected deaths. ${ }^{2}$

As the third most common cancer diagnosed in the USA for both men and women, colorectal cancer is also a societal

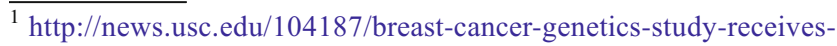
national-cancer-institute-grant/

${ }^{2} \mathrm{http} / / /$ www.cancer.org/cancer/pancreaticcancer/detailedguide/pancreaticcancer-key-statistics
} 
burden, with 135,000 new cases of colon cancer and 49,000 rectum cancer expected in $2016 .^{3}$ These two cancers represent $8.0 \%$ of all new cancer cases in the USA. The 5 year survival rate between 2006 and 2012 was estimated to be around $65 \%$.

In this issue, R. Goldschmeding et al. present new aspects of CCN biology in colorectal cancer. After reviewing the potential activities of CCN2 in tumor development, and the available colorectal cancer classifications, Goldschmeding et al. report and discuss their novel data which establish a clear association between $\mathrm{CCN} 2$ and colorectal cancer consensus molecular subtype 4 (CMS4). Interestingly, CMS4 is described as $23 \%$ mesenchymal with prominant TGFbeta overexpression and stromal invasion.

On a different standpoint, the review of normal hematopoiesis and related cancers provided by S. Irvine and colleagues highlights the implication of $\mathrm{CCN}$ proteins in hematopoiesis. The predicted values for $2016^{4}$ indicate that among 60,000 cases of expected patients with leukemia, 24,000 will not recover. Among these, 8200 cases of chronic myeloid leukemia of which 1000 will die, and 20,000 cases of acute myeloid leukemia with an expected death rate of $50 \%$. The regulatory role of the bone marrow microenvironment and various aspects of chemotherapy targeting hematopietic stem cells are discussed, with the potential use of $\mathrm{CCN}$ proteins in targeting both the microenvironment and malignant stem cells to improve therapeutic efficacy.

Other critical aspects of oncogenesis are addressed in this issue by M. Herlyn's group. In melanomas, $\mathrm{CCN} 1$ and $\mathrm{CCN} 3$ were reported to counteract metastasis, whereas the expression of CCN2 by tumor stroma was required for melanoma metastasis. Melanoma of the skin is a major societal issue due to the relationship that exists between sun exposure and tumor development. The predictions for $2016^{5}$ are 76,000 cases of which 10,000 will decease. In their review, Wang et al. address the role of cell-cell communication and CCN3 signaling, in human melanocyte homeostasis, and the implication of intercellular cell communication, with a particular emphasis on the Notch and Wnt pathways in melanocyte differentiation.

This special issue did not intend to cover all aspects of CCN-cancer relationships but only provide a flavor of some major topics in the field.

There are several other neoplasms in which the potential role of $\mathrm{CCN}$ proteins has been the studied. Amongst these, prostate carcinoma which is the most frequent cancer in human males after skin cancer appears to be a real societal burden with 180,000 new cases expected in 2016 among American men, of which 26,000 are expected to result in death. Current studies should allow more light to be shed on the implications of $\mathrm{CCN}$ proteins. Other cancers in which the role and/or implications have been studied include liver, lung, glioma and gastric cancers.

Along another line, the survey that we have performed, regarding the trends of $\mathrm{CCN}$ publication (A. Perbal and $\mathrm{B}$. Perbal in this issue), has confirmed that the $\mathrm{CCN}$ field is indeed growing at a very satisfactory pace with more than 4000 publications published since the early 90's. However, it has also highlighted the confusion and deleterious consequences which have stemmed from a disparate usage of various acronyms for each of the six members of the CCN family of proteins. We hope that the conclusions that we have drawn from our survey will help colleagues from all fields everywhere to unite and «play the same game».

We are delighted to open the columns of JCCS (note the IF 3.123 for 2015) to all those who wish to help build a strong and attractive $\mathrm{CCN}$ field full of translational medical promises for the near future. In this spirit, potential authors willing to contribute a review for JCCS are most welcome.

\footnotetext{
$\overline{3}$ see National Cancer Institute - Surveillance, Epidemiology and End Results Program - http://seer.cancer.gov/statfacts/

${ }^{4}$ ibid. note 3

${ }^{5}$ ibid. note 3
} 